\title{
Uso de Entrevistas na Avaliação de Jogos Baseados em Localização: Lições e Desafios
}

\author{
Nayana Carneiro \\ Universidade Federal do Ceará \\ Fortaleza, Ceará, Brasil \\ nayanacarneiro@great.ufc.br
}

\author{
Ticianne Darin \\ Universidade Federal do Ceará \\ Fortaleza, Brazil \\ ticianne@virtual.ufc.br
}

\author{
Windson Viana \\ Universidade Federal do Ceará \\ Fortaleza, Ceará, Brazil \\ windson@virtual.ufc.br
}

\section{RESUMO}

Games User Research (GUR) é um campo de estudo que tem buscado formar um conjunto de técnicas para avaliar a interação dos jogadores em diversos tipos de jogos. Buscando somar esforços aos avanços nessa área, este trabalho foca na avaliação da interação com jogos baseados em localização (JBL), buscando compreender quais aspectos da player experience em JBL podem ser explorados por meio de entrevistas e como isso tem sido feito pelas comunidades de prática e pesquisa. Para isso, foram analisados trabalhos que aplicaram o método em tal contexto e conduzido um survey de opinião com os respectivos autores. Como resultado, são apresentadas desafios de pesquisa e lições para o uso de entrevistas nesse tipo de avaliação, visando incentivar sua aplicação consciente e sistematizada, além de prover auxílio a praticantes e pesquisadores, especialmente iniciantes.

\section{PALAVRAS-CHAVE}

Jogos baseados em localização, Métodos de avaliação, Interação, Entrevistas

\section{INTRODUÇÃO}

Games User Research (GUR) é um campo de estudo advindo da Interação Humano-Computador (IHC) que procura analisar e entender a interação e a experiência de jogadores com jogos digitais, por meio da construção de evidência empírica, com o objetivo de melhorar todos os aspectos de um game design [18]. Para tal, a comunidade de GUR tem buscado formar um conjunto de técnicas e ferramentas específicas para avaliar essa interação [39], trabalhando com a aplicação e a

Permission to reproduce or distribute, in whole or in part, material extracted from this work, verbatim, adapted or remixed, as well as the creation or production from the content of such work, is granted without fee for noncommercial use, provided that the original work is properly credited.

IHC 2019 - Workshop sobre Interação e Pesquisa de Usuários no Desenvolvimento de Jogos (WIPlay), Outubro 21-25, 2019, Vitória, Brasil. In Anais Estendidos do XVIII Simpósio Brasileiro sobre Fatores Humanos em Sistemas Computacionais. Porto Alegre: SBC.

(c) 2019 by the author(s), in accordance with the terms of the Creative Commons Attribution-NonCommercial 4.0 International Public License (CC BY-NC 4.0). adequação de métodos de outras áreas para criar instrumentos mais adequados aos seus propósitos e responder questões relevantes sobre o comportamento humano e atitudes dos jogadores em diversos tipos de jogos [18].

Buscando somar esforços a esses avanços, o presente trabalho foca no estudo dos chamados jogos baseados em localização (JBL), um subtipo dos jogos pervasivos que faz uso de tecnologias de localização para integrar a posição de um ou mais jogadores às suas regras como um elemento central do jogo [26]. Essas informações são utilizadas para modificar seu estado em tempo de execução e criar uma conexão significativa entre o mundo real e o virtual, transformando o espaço físico no cenário de jogo [16]. Assim, os JBL possuem especificidades que os distinguem de outros jogos convencionais, como mobilidade, expansão espacial e temporal, pervasividade, e uso de informações contextuais $[1,26]$. Além de alcançar grande sucesso entre o público - exemplos disso são Pokémon Go (2016) e Harry Potter: Wizards Unite (2019) - esses jogos conquistaram espaço na indústria e despertaram o interesse da comunidade acadêmica [24], passando a ser aplicados em diferentes domínios (como saúde [12], educação [41] e turismo [3]), e trouxeram novos desafios de pesquisa, sendo um deles a avaliação da interação entre jogador e jogo e da experiência decorrida disso [9, 43].

Nesse contexto, os autores do presente trabalho iniciaram uma pesquisa com o objetivo final de prover um guia para avaliação qualitativa da interação com JBL que auxilie pesquisadores e praticantes. Para construir o conhecimento necessário a essa tarefa, foram realizadas duas fases da pesquisa. Na primeira, foi conduzido um mapeamento sistemático da literatura [27] que visava investigar como as comunidades de prática e pesquisa têm avaliado a interação dos jogadores com JBL, conforme reportado em [9]. Foram analisados 51 trabalhos que relatavam avaliar qualidades da interação entre jogador e jogo. Os resultados mostraram que player experience (PX) tem sido a qualidade da interação mais comumente avaliada e que a maior parte das estratégias utilizadas tem sido fortemente baseadas na aplicação de questionários, principalmente aqueles elaborados ad hoc.

Como já discutido na literatura (e.g., [18, 32]), questionários são extremamente úteis, contudo, oferecerem apenas 
uma visão geral do fenômeno estudado e feedback limitado. Por isso, sua aplicação isolada deve ser evitada em estudos que buscam investigações aprofundadas, uma vez que uma abordagem fundamentada no uso de múltiplos métodos é o caminho mais desejável $[14,32]$. Um dos métodos que a GUR tem utilizado para investigar em maior profundidade a PX e obter feedback rico dos jogadores é o de entrevistas [18]. Esse método oferece diversos benefícios para a pesquisa com usuários, sendo útil no contexto de avaliação da PX por ajudar a construir o entendimento das necessidades, preferências, motivações e atitudes dos jogadores [32]. Nos estudos de GUR, entrevistas são parte essencial de uma sessão qualitativa de testes com usuários [18].

Diante disso, o presente artigo relata a condução da segunda fase da pesquisa sobre avaliação da interação em JBL. O objetivo desta fase é aprofundar os resultados obtidos na Fase 1, para compreender quais aspectos dessa interação podem ser explorados por meio de entrevistas e como isso tem sido feito pela comunidade. Para tal, foram feitas análises adicionais de 23 trabalhos identificados no mapeamento que relataram utilizar entrevistas em suas avaliações e, posteriormente, foi conduzido um survey de opinião com os autores desses artigos, para investigar como eles têm utilizado o método no referido contexto e extrair orientações de suas experiências.

A contribuição deste artigo está na identificação de lições e desafios de pesquisa visando fomentar a discussão e reflexão acerca do uso de entrevistas na avaliação da interação com JBL, incentivando sua aplicação consciente e sistematizada. Além disso, as lições auxiliam a praticantes e pesquisadores especialmente os pouco experientes - em planejar e conduzir avaliações de JBL utilizando entrevistas.

\section{ENTREVISTAS E GAMES USER RESEARCH}

Entrevistas oferecem diversos benefícios para a pesquisa com usuários e são valiosas para fomentar a investigação aprofundada de determinados tópicos e esclarecer dúvidas com base nos feedbacks oferecidos pelos entrevistados [32]. Além de constituir um dos métodos de pesquisa mais utilizados em IHC $[32,53]$, entrevistas são úteis no contexto de avaliação da PX porque ajudam a construir o entendimento de necessidades, preocupações, preferências e atitudes dos jogadores [23], permitindo explorar questões mais complexas que as abordadas em surveys, por exemplo [52].

Nos estudos de GUR, entrevistas são consideradas parte essencial de uma sessão qualitativa de testes com usuários [18], pois oferecem um dos únicos modos de validar observações, descobrir problemas, coletar opiniões e encontrar causas para dificuldades enfrentadas pelos jogadores [18]. Além disso, podem ainda ser combinadas com outros métodos para enriquecer os dados coletados e criar uma visão holística do pensamento e comportamento do usuário, sendo parte primária na descoberta e compreensão de problemas de usabilidade e de contrariedades na experiência do jogador. Desse modo, entrevistas podem ser a escolha mais adequada a determinados objetivos de pesquisa e tipos de conhecimento almejados [23].

Uma vez que esse método possui a capacidade de revelar dimensões emocionais de uma experiência que nem sempre estão evidentes no comportamento de um indivíduo [30], diversos pesquisadores têm explorado seu potencial, elaborando novas técnicas e abordagens para aplicá-lo em diferentes áreas e contextos. Por exemplo, El-Nasr et al. [20] propuseram um método de avaliação formativa que usa de entrevistas retrospectivas para investigar como jogadores aceitam e integram um jogo em suas vidas - método particularmente adequado para pesquisas com jogos pervasivos em cenários naturalistas. Crawford, Monks e Wells [13] elaboraram uma técnica de entrevista baseada em realidade virtual para avaliar candidatos a residência em emergência médica e identificar suas habilidades de comunicação, resolução de problemas e trabalho em equipe. Mais recentemente, Holmes [22] tem explorado o método de entrevistas baseadas em brincadeiras, que permite observar e entrevistar crianças pequenas, levando em consideração suas limitações cognitivas e de linguagem, por meio de atividades lúdicas que promovam engajamento e comunicação direta entre entrevistador e entrevistado.

Esses estudos são apenas alguns exemplos da versatilidade e do potencial de uso das entrevistas. Contudo, é necessário destacar que seu processo de planejamento, condução e análise de dados é uma tarefa árdua e que deve ser feita de forma rigorosa. Apesar de diversos trabalhos oferecerem orientações e diretrizes de uso (e.g., [5, 29, 49, 55]), questões mais especificas hão de requerer cuidadosa consideração. Ironicamente, muitos pesquisadores e praticantes reduzem as entrevistas qualitativas a uma atividade trivial e não problemática, vendo-as como um meio fácil e garantido de se obter dados. Em consequência disso, muitos artigos reportam a aplicação do método de modo inadequado e superficial, como identificado por Myers e Newman [38] em pesquisas na área de Sistemas de Informação (SI). Certamente essa deficiência não é exclusividade da área e pode exemplificar questões que ainda precisam ser analisadas e discutidas também no domínio de jogos - como a adequação do método, seus usos e a falta de rigor na aplicação, análise e reporte. O presente trabalho investiga parte desses tópicos, compondo um passo inicial na busca pelo tratamento de tais práticas.

\section{METODOLOGIA}

Na primeira fase desta pesquisa, foi conduzido um mapeamento sistemático da literatura com o objetivo de investigar como as comunidades de prática e pesquisa têm avaliado qualidades da interação entre jogador e JBL. O mapeamento 
seguiu a abordagem proposta por Kitchenham, Budgen e Brereton [27], e visava responder três questões de pesquisa: (QP1) Quais métodos são utilizados na avaliação de $7 B L$ ?; (QP2) Quais qualidades da interação são avaliadas?; (QP3) Quais estratégias e abordagens são empregadas nesse tipo de avaliação? A busca por trabalhos foi feita em cinco bases (Scopus, ACM Digital Library, Web of Science, IEEE Xplore e Science Direct) usando a string de busca apresentada na Figura 1. Como resultado, obteve-se um conjunto inicial de 437 trabalhos que passaram por um processo de filtragem em três passos, resultando no conjunto final de 51 artigos, dos quais foram extraídas informações para responder às questões de pesquisa (mais detalhes em [9]). A Tabela 1 apresenta os principais critérios de inclusão e exclusão utilizados.

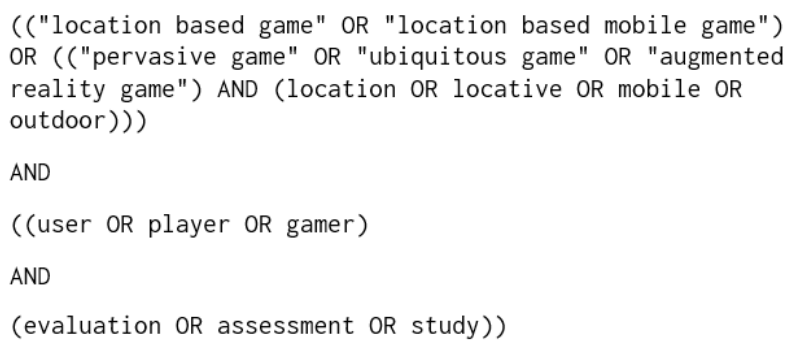

Figura 1: String submetida às bases de dados

Tabela 1: Principais critérios de inclusão e exclusão

\begin{tabular}{cl}
\hline \multirow{2}{*}{ Inclusão } & $\begin{array}{l}\text { O estudo foca em JBL E Reporta a } \\
\text { avaliação de qualidades da interação entre } \\
\text { jogador e jogo } E \text { É um estudo primário }\end{array}$ \\
Exclusão estudo não foca em JBL OU Não avalia \\
$\begin{array}{l}\text { qualidades da interação } O U \text { O trabalho } \\
\text { não está escrito em Inglês ou Português }\end{array}$ \\
\hline
\end{tabular}

Observou-se que player experience é a qualidade da interação mais frequentemente avaliada, e aplicação de survey, o método mais utilizado na avaliação de JBL, seguido de entrevista e registro de logs de interação. No entanto, os resultados direcionaram o foco da pesquisa para um exame mais detalhado da utilização de entrevistas na avaliação de JBL, uma vez que a análise inicial dos trabalhos deu indícios de que esse método têm sido pouco explorado de modo sistemático e é frequentemente visto como uma atividade que requer pouco rigor em sua aplicação e análise. Assim, na segunda etapa da pesquisa, foram feitas análises quantitativas adicionais para identificar relacionamentos entre os dados especificamente nos 23 trabalhos (extraídos do conjunto final de 51) que reportavam utilizar entrevistas e, posteriormente, foi aplicado um survey de opinião com os autores dos referidos artigos.

\section{Survey de Opinião com Especialistas}

Surveys de opinião visam determinar o que os participantes pensam sobre certos conceitos [42] e, quando conduzidos com especialistas, podem servir a vários propósitos, sendo úteis para identificar problemas, prever mudanças e esclarecer questões relevantes em um tópico específico [15, 48], por exemplo. Diante disso, foi elaborado um formulário on-line com o objetivo de aprofundar a compreensão de como os autores dos 23 trabalhos aplicaram entrevistas para avaliar a interação com JBL em seus estudos, visando identificar lacunas e extrair lições de suas experiências com o método.

O convite para participação no survey foi enviado por email e, no caso de autores cujos endereços eletrônicos não foram encontrados, foi feito por meio da rede social Research Gate $^{1}$. Seis autores foram excluídos da lista de envio por falta de contatos válidos, totalizando 81 destinatários. Cada autor recebeu uma mensagem personalizada, contendo o título do artigo identificado no mapeamento e uma breve explicação da pesquisa com um hyperlink de acesso ao formulário. Alguns autores constavam em mais de um trabalho e, por isso, receberam um formulário diferenciado. Passados dez dias do primeiro envio, foram emitidos lembretes aos autores. O formulário ficou disponível por aproximadamente dois meses e foi encerrado após a estagnação do número de respostas.

O formulário possuía 17 perguntas (nove objetivas e oito subjetivas) divididas em três seções: (i) perfil do respondente, (ii) questões sobre o uso de entrevistas na avaliação do JBL no respectivo estudo, e (iii) sondagem quanto à abertura dos participantes a uma proposta de adaptação do método para o contexto de avaliação de JBL. Catorze autores, responsáveis por 11 trabalhos, participaram do survey, significando uma taxa de resposta de $17,28 \%(\mathrm{~N}=14)$. Apesar de ser uma parcela relativamente pequena de respondentes, a amostra obtida condiz com recomendações na literatura que indicam o uso de grupos de cinco a 20 especialistas [48]. Ademais, os participantes responderam ao survey de modo independente e possuem backgrounds diversos (atuando em diferentes países, por exemplo, Reino Unido, Grécia, México e Indonésia), o que reduz o potencial de tendências específicas nas respostas [15]. A análise das respostas foi feita por meio da identificação de temas recorrentes [47]: os dados foram tabulados, agrupados e codificados de acordo com tópicos que emergiram dos próprios dados. A partir dos códigos, foram criadas categorias que foram sistematicamente analisadas e, posteriormente, deram origem às lições listadas na Seção 5.

\section{RESULTADOS E DISCUSSÃO}

Nesta seção, são apresentados e discutidos os resultados da $2^{\text {a }}$ fase da presente pesquisa, composta por análises quantitativas dos 23 trabalhos identificados no mapeamento que

\footnotetext{
${ }^{1}$ https://www.researchgate.net/
} 
utilizaram entrevistas em suas avaliações e de um survey de opinião com os autores dos referidos artigos.

\section{Uso de Entrevistas no Mapeamento Sistemático}

Foram identificados $21 \mathrm{JBL}$ avaliados pelos 23 trabalhos, conforme mostra a Tabela 2 . A maior parte $(80,95 \%)$ dos jogos avaliados foi desenvolvida para fins de pesquisa - apenas três $(14,29 \%)$ eram comerciais. Oito $(38,10 \%)$ estudos avaliaram protótipos funcionais, dez $(47,62 \%)$ avaliaram versões para teste, e três $(14,29 \%)$ usaram versões disponíveis no mercado. Três $(14,29 \%)$ JBL tinham propósitos educacionais, enquanto $18(85,71 \%)$ visavam o entretenimento. No restante desta subseção são discutidas questões relacionadas à forma de avaliação destes jogos. É importante ressaltar, no entanto, que os resultados relacionados às qualidades avaliadas e aos tamanhos da amostra se referem a todos os métodos empregados em cada avaliação, não necessariamente somente às entrevistas.

Número de participantes. O tamanho das amostras utilizadas nos estudos variou de 6 a 96 usuários. A média de participantes foi de 24,56 usuários por estudo (D.P. = 19,72), e as amostras mais frequentemente usadas eram formadas por 10 ou 24 participantes (relatadas em 3 estudos cada). Os trabalhos que apresentaram as maiores amostras reuniram 36 [40], 60 [6] e 96 [51] participantes.

Qualidades avaliadas. Foram identificadas 20 qualidades da interação sob avaliação, com uma média de 1,87 qualidades por artigo (D.P. = 1,14). Player experience foi a mais frequentemente avaliada (indicada em 14 trabalhos), seguida por usabilidade, imersão e presença (avaliadas em quatro trabalhos cada). Outras qualidades menos avaliadas foram engajamento, jogabilidade e presença espacial, conforme mostra a Figura 2. A maior parte dos estudos (12, i.e., 52,17\%) focou em avaliar apenas uma qualidade - em sete deles, essa qualidade foi PX. O trabalho de McCall e Braun [35] avaliou o maior número de qualidades (cinco), sendo elas: usabilidade, PX, presença, sense of place e presença social. Dos 14 trabalhos que avaliaram PX, sete também se propuseram a avaliar outras qualidades, sendo elas usabilidade, engajamento, imersão, enjoyment e presença. Vale destacar que, frequentemente na literatura, algumas dessas qualidades (e.g., presença e imersão) são abordadas como componentes da PX, ou ainda, parte do conjunto de propriedades que descrevem essa experiência, como afirmam Sánchez et al. [50], por exemplo. No entanto, esses trabalhos parecem tratar PX e as referidas qualidades como aspectos distintos, sem discutir tal questão.

Métodos e estratégias de avaliação. Além de entrevista, foram identificados outros 10 métodos aplicados nos estudos, como mostra a Figura 3. Três $(13,04 \%)$ trabalhos utilizaram um único método (i.e., entrevista), enquanto os outros $20(86,96 \%)$

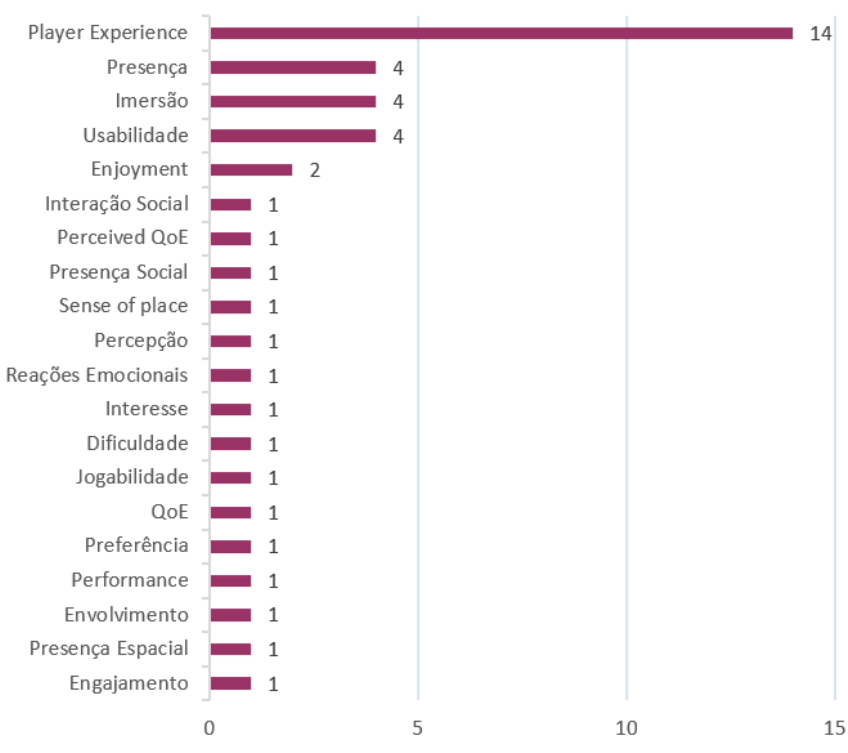

Figura 2: Qualidades da interação sob avaliação nos trabalhos analisados

seguiram abordagens de múltiplos métodos [14, 32]. Os três estudos que usaram unicamente entrevistas [19, 34, 46] avaliaram a PX em jogos desenvolvidos para pesquisa (Ekman [19] avaliou também jogabilidade), dois deles em protótipos funcionais [19, 34] e um em versão para teste [46]. Os outros $20(86,96 \%)$ estudos combinaram entrevistas com pelo menos mais um método: questionário foi o mais frequente nas combinações, sendo usado em 18 (78,26\%) trabalhos. Observação de uso foi o segundo mais aplicado (14 estudos, 60,87\%), seguindo uma tendência comum de vincular entrevistas a algum tipo de observação [30]. Vale destacar que apenas dois $(8,70 \%)$ estudos $[10,56]$ utilizaram monitoramento de dados fisiológicos - ambos aplicaram também entrevista, observação e registro de $\log s$ de interação. Uma possível explicação para a baixa utilização desses métodos é que a avaliação de JBL, idealmente, é feita em ambientes externos ou que simulem contextos de mobilidade, o que pode dificultar o uso de alguns dos equipamentos que capturam essas medidas. É possível também que dificuldades vinculadas ao contexto expliquem o fato de que apenas um trabalho [40] usou Think Aloud [21]. Esses casos ilustram alguns dos desafios que acompanham esse tipo de avaliação.

Análise de dados. Apesar de todos os estudos terem aplicado entrevistas, catorze $(60,87 \%)$ artigos não informaram como (ou se) foi feita a análise dos dados qualitativos. Desses, três $(13,04 \%)$ não mencionaram a realização de nenhum tipo de análise, seja quantitativa ou qualitativa - dois desses aplicaram apenas entrevistas $[19,46]$. Os demais nove $(39,13 \%)$ trabalhos descreveram o processo de análise com 
Tabela 2: JBL avaliados nos trabalhos analisados

\begin{tabular}{cccccl}
\hline Jogo & Trabalho & Jogo & Trabalho & Jogo & Trabalho \\
\hline Barbarossa & {$[25]$} & Ghost Detector & {$[40]$} & Parallel Kingdom & {$[56]$} \\
Block!Block! & {$[31]$} & GlowPhones & {$[37]$} & Pokémon GO & {$[45]$} \\
Can You See Me Now? & {$[4]$} & Growl Patrol & {$[28]$} & Spellbound & {$[54]$} \\
Cutting Corners & {$[51]$} & Horror game & {$[44]$} & Shhh! & {$[34]$} \\
Destination & {$[2]$} & Knowledge War & {$[57]$} & SoundPacman & {$[10]$} \\
Epidemic Menace & {$[33]$} & LOCUNET game & {$[17]$} & The Songs of North & {$[19]$} \\
Fruit Farmer & {$[8]$} & Obscura & {$[46]$} & TimeWarp & {$[6,35,36]$} \\
\hline
\end{tabular}

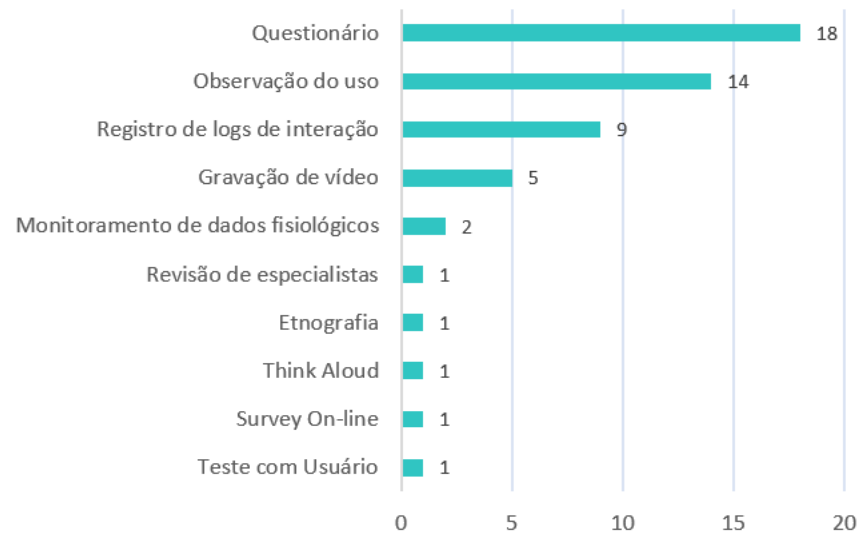

Figura 3: Métodos utilizados em combinação com entrevistas nas avaliações relatadas pelos trabalhos analisados

níveis de detalhamento variados e, em alguns casos, de modo superficial. Apenas três artigos mencionaram análises qualiquantitativas, enquanto os outros indicaram somente as análises qualitativas ( 3 trabalhos) ou somente as quantitativas (3 trabalhos). Algumas técnicas mencionadas foram: análise temática indutiva, codificação com esquema próprio e diagrama de afinidade. Dois trabalhos [17, 34] se destacaram por prover mais detalhes sobre os procedimentos de análise qualitativa dos dados obtidos em entrevistas.

\section{Visão Geral dos Resultados do Survey}

Perfil dos autores. O survey recebeu respostas de 14 autores, provenientes de oito países: Alemanha, Áustria, Canadá, Dinamarca, Grécia, Indonésia, México e Reino Unido. Doze $(85,71 \%)$ deles atuam na área de IHC, sete (50\%) em Game Design e seis $(42,86 \%)$ trabalham também com JBL. Apenas três $(21,43 \%)$ listaram GUR entre suas áreas de atuação. Sete (50\%) participantes trabalham com avaliação de jogos há 10 anos ou mais, cinco $(37,71 \%)$ possuem entre 1 e 6 anos de experiência, e dois $(14,29 \%)$ utilizam esse tipo de avaliação em suas pesquisas apenas esporadicamente e não a consideram uma de suas áreas de atuação. Seis $(42,82 \%)$ autores indicaram conhecimento de guidelines da literatura sobre elaboração e condução de entrevistas - os trabalhos de Steinar Kvale [29] e Larry Wood [58] foram os mais indicados -, no entanto, o uso delas não foi mencionado em nenhum dos artigos. A Tabela 3 resume os perfis dos participantes.

Motivações para usar entrevistas. As principais razões apontadas foram a busca por uma compreensão mais aprofundada das qualidades avaliadas e por feedback rico e detalhado. Outro motivo comum foi a consciência de que as entrevistas poderiam complementar os dados obtidos com os métodos quantitativos, especialmente quando o objetivo era investigar percepções, comportamentos e emoções dos usuários. Para o especialista A13, as entrevistas permitiram escrutinar padrões de comportamento potencialmente interessantes observados durante os testes de usabilidade. Outros motivos listados foram flexibilidade, contato direto com os usuários, coleta sistemática de feedback e o uso de dados da entrevista como guia para interpretar os dados quantitativos. $\mathrm{O}$ autor A11 disse ter escolhido entrevista semiestruturada por ser um método rápido e fácil de aplicar em ambientes externos. Consoante a isso, A6 destacou que o uso de entrevista permitiu investigar mais detalhadamente pontos críticos observados nos testes por oferecer mais liberdade aos participantes, o que gerou informações valiosas que não seriam obtidas com outros métodos. Uma vez que incidentes críticos podem trazer consequências reais aos jogadores de JBL, essas condições ganham extrema relevância nesse tipo de avaliação.

Elaboração do roteiro. Todos os autores disseram ter aplicado entrevistas semiestruturadas nos referidos estudos. A principal abordagem para composição do roteiro foi a elaboração pelos próprios autores, tendo como base os objetivos da avaliação e do estudo. Outras práticas comuns foram criar o roteiro a partir de discussões entre os coautores, e deixar os colegas com maior experiência responsáveis por essa atividade. Dois autores adaptaram roteiros de outros estudos e um afirmou ter utilizado uma consultoria em usabilidade para criar o roteiro, visto que avaliação de jogos não é sua área de atuação. Apenas três autores seguiram diretrizes para aplicação de entrevistas propostas na literatura - contudo, apenas um trabalho [25] relatou isso. Desse modo, a maior 
Tabela 3: Perfil dos autores participantes do survey

\begin{tabular}{|c|c|c|c|c|c|}
\hline ID & Origem & $\begin{array}{c}\mathrm{N}^{\circ} \text { de } \\
\text { artigos }\end{array}$ & $\begin{array}{l}\text { Experiência } \\
\text { av. de Jogos }\end{array}$ & Áreas de atuação & $\begin{array}{l}\text { Familiaridade com } \\
\text { guidelines }\end{array}$ \\
\hline A1 & Áustria & 1 & $\geq 10$ anos & IHC, Game Design & Nenhuma das citadas \\
\hline A2 & Alemanha & 2 & $\geq 10$ anos & $\begin{array}{c}\text { IHC, Game Design, Computação Móvel, Jogos } \\
\text { Pervasivos, JBL }\end{array}$ & Não familiar \\
\hline A3 & Canadá & 1 & $\geq 10$ anos & IHC, Game Design, Gamificação & Wood [58] \\
\hline A4 & México & 1 & $\geq 10$ anos & IHC, GUR & Nenhuma das citadas \\
\hline A5 & Indonésia & 1 & 4 a 6 anos & $\begin{array}{l}\text { IHC, Métodos de Avaliação, Game Design, GUR, } \\
\text { Gamificação, JBL }\end{array}$ & Kvale [29], Boyce [7] \\
\hline A6 & Grécia & 1 & 4 a 6 anos & $\begin{array}{l}\text { IHC, Mét. de Avaliação, Game Design, GUR, } \\
\text { Gamificação, Comp. Móvel e Ubíqua, JBL }\end{array}$ & Nenhuma das citadas \\
\hline A7 & Reino Unido & 1 & $\mathrm{~N} / \mathrm{A}$ & Entrevistas Investigativas & Nenhuma das citadas \\
\hline A8 & Reino Unido & 1 & $\geq 10$ anos & Informação e Inteligência Geográfica & Wood [58] \\
\hline A9 & Reino Unido & 1 & 1 a 3 anos & IHC, Métodos de Avaliação & $\begin{array}{l}\text { Kvale [29], Myers e } \\
\text { Newman [38], } \\
\text { Chauncey [11] }\end{array}$ \\
\hline A10 & Reino Unido & 1 & $\geq 10$ anos & IHC, Gamificação, JBL & Nenhuma das citadas \\
\hline A11 & Dinamarca & 1 & 1 a 3 anos & $\begin{array}{c}\text { IHC, Game Design, Gamificação, Computação } \\
\text { Móvel, JBL }\end{array}$ & Kvale [29] \\
\hline A12 & Reino Unido & 1 & $\mathrm{~N} / \mathrm{A}$ & $\mathrm{IHC}$ & Nenhuma das citadas \\
\hline A13 & Reino Unido & 1 & 1 a 3 anos & IHC, Computação Ubíqua & Wood [58] \\
\hline A14 & Reino Unido & 1 & $\geq 10$ anos & IHC, Game Design, Comp. Móvel e Ubíqua, JBL & Nenhuma das citadas \\
\hline
\end{tabular}

parte dos autores demonstrou confiar em suas experiências prévias com o método, nas discussões com os colegas e no uso dos objetivos da pesquisa como parâmetro para criar as perguntas. Apenas dois autores (A11 e A14) relataram dificuldades na elaboração do roteiro, sendo elas: reunir e abordar todos os assuntos relevantes para o estudo, e compor um roteiro sólido com bom equilíbrio entre estrutura e flexibilidade de modo que o entrevistador possa seguir o fluxo dos usuários (o que costuma produzir insights inesperados), mas ainda obter bons comparadores entre os dados.

Dificuldades na aplicação do método. Cinco autores relataram dificuldades na condução das entrevistas, destacando adversidades inerentes aos contextos de realização das avaliações (por exemplo, baixas temperaturas durante os testes em espaço aberto). Uma dificuldade comum é que, por estarem em um ambiente externo, os participantes ficam mais sujeitos a distrações e frequentemente precisam ser conduzidos de volta às questões da entrevista. Paralelo a isso, A6 relatou que, apesar de os entrevistadores buscarem feedback profundo sobre a PX, os usuários tendiam a falar muito mais de suas opiniões sobre o visual do jogo, sendo difícil levá-los ao cerne das perguntas. Um dos autores mais experientes, A14, apontou o desafio de se estabelecer uma comunicação clara e efetiva entre entrevistador e entrevistado, principalmente quando o avaliador busca coletar informações "técnicas" de usuários que possuem pouca compreensão do assunto, e destacou a importância de um entrevistador treinado para situações como essa.

Especificidades das entrevistas para avaliar JBL. Dez (71,43\%) autores consideraram questões relacionadas a, pelo menos, uma especificidade dos JBL para elaborar o roteiro das entrevistas. De modo geral, a localização foi apontada como o aspecto mais explorado nos estudos, seguida por mobilidade e interação social no jogo. A ênfase nessas especificidades 
se alinha às características mais comumente atribuídas aos JBL na literatura - por exemplo, De Souza e Sutko [16] apontam localização, mobilidade e sociabilidade como as principais características desses jogos. Outras especificidades consideradas foram sensibilidade ao contexto, conectividade, pervasividade, segurança, privacidade e imprecisão do GPS As menos exploradas foram expansão temporal e espacial - apesar de ambas constarem na literatura como aspectos essenciais dos JBL [1] - e nível de esforço físico. Com relação ao reaproveitamento do roteiro utilizado para avaliar os JBL nos estudos, a maioria dos autores $(85,7 \%)$ acredita que não poderia reutilizá-lo para avaliar outros tipos de jogos, uma vez que o foco da entrevista depende fortemente dos objetivos estabelecidos para a pesquisa. Outro ponto levantado foi que os roteiros usados nos referidos estudos eram bastante específicos, sendo aplicáveis apenas ao jogo e contexto em questão. Dois autores (A1 e A6) disseram que reutilizariam seus roteiros, fazendo pequenos ajustes para incluir aspectos contextuais dos JBL e questões particulares à qualidade da interação sob avaliação. A6 destacou que, apesar de "jogar um JBL" ser indiscutivelmente diferente de "jogar um jogo convencional em um smartphone", é possível usar instrumentos semelhantes para avaliar determinados aspectos em ambos os jogos (e.g., grau de realismo dos gráficos). No entanto, quando o foco da avaliação repousa em questões mais subjetivas, como PX, é imprescindível considerar as particularidades de cada jogo, pois elas ditarão as mudanças a serem feitas nos roteiros das entrevistas para cada caso.

\section{LIÇÕES E DESAFIOS PARA A AVALIAÇÃO DE JBL}

De modo geral, os especialistas atestaram os benefícios do uso de entrevistas em seus estudos. Assim, os resultados obtidos foram traduzidos em lições para a condução desse tipo de avaliação. Essas informações foram extraídas das respostas dos especialistas ao survey, mas também são frutos do mapeamento, das análises adicionais e de conhecimentos obtidos em estudos da literatura. Juntas, elas formam um conjunto inicial de orientações que visa munir a comunidade de prática e pesquisa, especialmente iniciantes, com recursos para a execução consciente de tal atividade. Também foram identificados desafios que refletem oportunidades de pesquisa, especialmente para as comunidades de IHC e GUR, e são apresentados como insights a serem discutidos (e posteriormente aprimorados e expandidos), visando o amadurecimento da avaliação de JBL.

Cinco das lições apresentadas podem ser aplicadas à avaliação de jogos e uso de entrevistas de modo geral (L4, L6, L8, L10, L12), enquanto as demais são específicas para JBL (L1, L2, L3, L5, L7, L9, L11). Vale destacar que a adequação das informações listadas deve ser julgada com base nas particularidades de cada pesquisa e estágios do processo de design de um JBL, não se aplicando igualmente a todos os casos.

\section{Dividir para conquistar: em busca de estratégias melhores para avaliar JBL}

Os resultados obtidos destacaram a importância da combinação de diferentes métodos, como já recomendado na literatura $[14,32]$. Como esperado, os autores indicaram adotar essa prática nas avaliações dos JBL, considerando-a fundamental, uma vez que a aplicação isolada de um único método não costuma prover as informações almejadas. Também ficou claro que nem todos os métodos podem ser aplicados igualmente nesse tipo de avaliação - pelo menos não sem alguma adaptação -, já que as características de um JBL e seu contexto de uso podem impor diversas restrições aos avaliadores. É preciso considerar essas questões ao planejar as estratégias de avaliação, mantendo o foco nos objetivos do estudo, qualidades da interação a serem avaliadas e características do(s) jogo(s). Algumas lições relacionadas são:

L1: As particularidades dos JBL impactam e distinguem a PX nesses jogos. Identifique e entenda as características marcantes do JBL avaliado para traçar estratégias que considerem e explorem essas questões. Escolha e adapte os métodos de coleta de dados tendo em mente que essas especificidades influenciarão na condução da avaliação e nos resultados obtidos.

L2: Combine métodos quantitativos e qualitativos, complementando suas limitações, mas evite aplicar métodos que limitem a vivência do jogador com as especificidades do JBL avaliado (e.g., uso de equipamentos que limitem a mobilidade do jogador).

L3: Priorize a avaliação em ambientes externos, incluindo e explorando as dificuldades inerentes a esse contexto (e.g., condições climáticas, distrações, imprecisão do GPS). Esses fatores fazem parte da experiência real de jogo em JBL e devem ser considerados em uma avaliação que busque uma visão realista da PX.

Desafio: Como planejar estratégias de avaliação que explorem adequada e sistematicamente as características e especificidades do $\mathrm{FBL}$ avaliado?

\section{Conhecer para aplicar: como potencializar o planejamento e uso de entrevistas para avaliar JBL?}

O uso de entrevistas costuma apresentar diferentes desafios aos avaliadores. Quando aplicadas à avaliação de JBL, um contexto muitas vezes imprevisível, questões comuns podem ganhar novas nuances, o que aumenta a importância de elaborar roteiros precisos e eficientes, mas que ainda ofereçam a entrevistador e entrevistado flexibilidade e liberdade. Além disso, para utilizar esse método da melhor forma e garantir que os objetivos sejam alcançados, é preciso conhecer suas potencialidades e limitações, para então planejar seu uso adequado e executá-lo de modo realmente proveitoso. 
L4: Entrevistas semiestruturadas são indicadas para avaliar a PX em JBL, pois oferecem flexibilidade e liberdade para que o usuário se expresse, enquanto permitem ao entrevistador aproveitar oportunidades e investigar mais a fundo pontos específicos, mantendo alguma estrutura que viabilize a coleta sistemática de dados.

L5: Ao elaborar o roteiro para a entrevista, tenha como foco os objetivos da avaliação, mas também priorize o exame de questões específicas dos JBL - como mobilidade, espacialidade, expansão temporal - e suas influências na PX (e.g., Como o esforço físico afeta a diversão do usuário?).

L6: Identifique quais objetivos da avaliação podem ser atingidos através de outros métodos e explore na entrevista aqueles que demandem exame mais profundo (por exemplo, questões subjetivas e esclarecimento de dúvidas). Algumas das vantagens de entrevistas para avaliar JBL são praticidade e rapidez, então seja objetivo e procure simplificar a condução do método.

Desafios: Como simplificar e estruturar a aplicação de entrevistas para a avaliação de $7 B L$ ? Como criar guidelines adequadas, atrativas e acessíveis aos praticantes dessa atividade?

\section{Entrevistas servem a múltiplos propósitos: é importante tirar proveito disso na avaliação de JBL}

Diversas motivações foram apontadas para a aplicação de entrevistas na avaliação de JBL. Versatilidade, flexibilidade e (relativa) rapidez na aplicação, por exemplo, tornam esse método valioso para avaliações realizadas em ambientes externos. Seguindo as tendências dos estudos de GUR, entrevistas se mostraram uma ferramenta particularmente valiosa para a avaliação de JBL, especialmente na investigação da PX, por atenderem a diversos fins e fornecerem dados que podem ser usados de diferentes modos (por exemplo, para guiar a interpretação de dados quantitativos), como os especialistas indicaram em suas respostas. Algumas lições obtidas são:

L7: Use entrevistas para investigar como as particularidades do JBL afetam a PX na visão do jogador. Busque coletar informações detalhadas sobre como ele percebe e lida com as características do jogo e suas especificidades (e.g., segurança, esforço, imagem social). Isso pode auxiliar na obtenção de dados que, dentre outros, podem gerar guidelines de design para esses jogos.

L8: Combine entrevistas com observação de uso, por exemplo, para investigar eventos críticos (inclusive os causados por fatores externos) e compreender como os usuários percebem, interpretam e reagem a eles. Esses eventos podem transformar a forma como o jogador interage com o JBL, criando dinâmicas de jogo que não haviam sido previstas pelos game designers.
L9: Use entrevistas para investigar como os jogadores integram o JBL à sua vida e qual o impacto disso na perspectiva e experiência do jogador. Uma vez que JBL são jogados em meio a atividades da vida diária, informações como essas são importantes para melhorar a PX e a dinâmica do jogo.

Desafio: Como garantir a coleta sistemática de informações significativas sem comprometer a flexibilidade do método?

\section{Com grandes poderes, grandes responsabilidades: entrevistas custam caro, mas valem o preço}

Entrevistas são um método eficaz na avaliação da interação entre jogador e JBL - 92,86\% dos especialistas confirmaram a eficácia do método para alcançar seus objetivos. $\mathrm{O}$ uso de entrevistas pode resultar em diversos benefícios para o estudo, como contato direto com os usuários, obtenção de feedback que não seria captado com outros métodos, maior compreensão das percepções dos jogadores e suporte na interpretação e verificação dos dados coletados com métodos quantitativos. No entanto, entrevistas não devem ser vistas como um modo fácil e garantido de se obter bons resultados. Seu uso adequado requer esforço e rigor, além de ser acompanhado de limitações e dificuldades, como qualquer outro método. Como atestado pelos especialistas, entrevistas demandam planejamento, preparação e podem resultar em feedback ruim, se conduzidas de modo frívolo. Além disso, a análise dos dados costuma ser demorada e laboriosa.

L10: Os entrevistadores devem ser familiares com as particularidades e características do JBL avaliado, assim eles serão capazes de reconhecer e explorar oportunidades nas falas e ações dos participantes que permitam investigar melhor a PX oferecida pelo jogo.

L11: Os JBL permitem grande liberdade aos jogadores, o que aumenta a variabilidade da PX. Apesar disso, é importante que a coleta de dados seja feita de forma sistemática para garantir bons parâmetros de comparação. Ao entrevistar um usuário, faça perguntas sobre aspectos da experiência que sejam comuns a todos os jogadores - as características do JBL avaliado podem gerar tópicos a serem consistentemente explorados, por exemplo.

L12: Combine entrevistas com métodos que capturem dados durante o jogo e explore "hightlights" do gameplay na perguntas da entrevista para obter resultados mais significativos. Durante a análise, esses dados podem ser cruzados e utilizados para "provar" as falas dos usuários com suas ações.

Desafio: Como tornar o processo de análise mais prático e direto para que entrevistas sejam aplicadas em contextos que exigem maior rapidez, como na indústria? 


\section{CONCLUSÃO E TRABALHOS FUTUROS}

Avaliar a interação de um jogador com um JBL ainda é uma tarefa complexa, dadas as características peculiares desses jogos. Diante disso, o presente trabalho relatou a segunda etapa de uma pesquisa que visa contribuir para a estruturação desse tipo de avaliação. Foram reportados resultados da análise de 23 trabalhos que utilizaram entrevistas para avaliar JBL, e da condução de um survey de opinião com os respectivos autores, visando identificar práticas comuns e traduzi-las em direcionamentos acessíveis a pesquisadores e praticantes interessados. A partir dos conhecimentos obtidos, foram elencados lições e desafios de pesquisa - com ênfase no uso de entrevistas - que apontam para a importância de considerar as especificidades dos JBL e investigar seus efeitos na interação ao avaliar a IHC nesses jogos. As informações apresentadas são úteis para auxiliar avaliadores nessa tarefa e incentivá-los a refletir e atuar em cima dessas questões.

No tocante ao uso de entrevistas, também foi observado que, apesar de haver na literatura diversas diretrizes sobre o método, uma parcela mínima dos especialistas afirmou ter utilizado tal auxílio nos trabalhos analisados. Esse fato não permite colocar em "xeque" a qualidade das entrevistas feitas pelos demais autores, mas abre espaço para indagações acerca dessa prática na avaliação de JBL. Uma vez que a comunidade científica se ocupa de preocupações que extrapolam questões apenas práticas, parece relevante investigar também as razões por trás desse modus operandi. É possível que as diretrizes existentes pareçam distantes do contexto de JBL, ou ainda, que a avaliação de IHC esteja sendo tratada como algo secundário nesses estudos. É preciso investigar questões como essas para promover a estruturação da área e oferecer recursos adequados à condução de avaliações mais acuradas e expressivas nesse domínio.

Desse modo, nos trabalhos futuros, pretende-se amadurecer e expandir as lições listadas e, posteriormente, formar um guia para avaliação qualitativa da PX em JBL, para orientar o planejamento e condução dessas avaliações de modo consistente e estruturado. Quando questionados sobre tal proposta, os especialistas a consideraram positiva, especialmente para benefício de estudantes e praticantes iniciantes. Assim, serão continuados os esforços nessa direção.

\section{REFERÊNCIAS}

[1] Ola Ahlqvist. 2018. Location-Based Games. International Encyclopedia of Geography: People, the Earth, Environment and Technology (2018), 1-6.

[2] Lynne Baillie, Lee Morton, Stephen Uzor, and David C Moffatt. 2010. An investigation of user responses to specifically designed activities in a multimodal location based game. Journal on Multimodal User Interfaces 3, 3 (2010), 179-188.

[3] Rafael Ballagas, André Kuntze, and Steffen P Walz. 2008. Gaming tourism: Lessons from evaluating rexplorer, a pervasive game for tourists. In International Conference on Pervasive Computing. Springer, 244-261.
[4] Steve Benford, Andy Crabtree, Martin Flintham, Adam Drozd, Rob Anastasi, Mark Paxton, Nick Tandavanitj, Matt Adams, and Ju RowFarr. 2006. Can you see me now? ACM Transactions on ComputerHuman Interaction (TOCHI) 13, 1 (2006), 100-133.

[5] Ann Blandford, Dominic Furniss, and Stephann Makri. 2016. Qualitative HCI Research: Going Behind the Scenes. Morgan \& Claypool Publishers.

[6] Lisa Blum, Richard Wetzel, Rod McCall, Leif Oppermann, and Wolfgang Broll. 2012. The final TimeWarp: using form and content to support player experience and presence when designing location-aware mobile augmented reality games. In Proceedings of the designing interactive systems conference. ACM, 711-720.

[7] Carolyn Boyce and Palena Neale. 2006. Conducting in-depth interviews: A guide for designing and conducting in-depth interviews for evaluation input. (2006)

[8] Eduardo Calvillo-Gámez, Jodi Crisp, and Pablo Romero. 2011. Empirical validation of the involvement component of the pervasive GameFlow model. In Proceedings of the 8th International Conference on Advances in Computer Entertainment Technology. ACM, 48.

[9] Nayana Carneiro, Ticianne Darin, and Windson Viana. 2019. What Are We Talking About When We Talk AboutLocation-Based Games Evaluation? A Systematic Mapping Study. In Proceedings of the 18th Brazilian Symposium on Human Factors in Computing Systems. ACM, 13.

[10] Thomas Chatzidimitris, Damianos Gavalas, and Despina Michael. 2016. SoundPacman: Audio augmented reality in location-based games. In 18th Mediterranean Electrotechnical Conference (MELECON). IEEE, 1-6.

[11] Wilson Chauncey. 2014. Interview Techniques for UX Practitioners. A User-Centered Design Method (2014).

[12] Luca Chittaro and Riccardo Sioni. 2012. Turning the classic snake mobile game into a location-based exergame that encourages walking. In International Conference on Persuasive Technology. Springer, 43-54.

[13] Scott B Crawford, Stormy M Monks, and Radosveta N Wells. 2018. Virtual reality as an interview technique in evaluation of emergency medicine applicants. AEM education and training 2, 4 (2018), 328-333.

[14] John W Creswell and Cheryl N Poth. 2018. Qualitative inquiry and research design: Choosing among five approaches. Sage publications.

[15] Ticianne Darin, Rossana Andrade, and Jaime Sánchez. 2019. Principles for Evaluating Usability in Multimodal Games for People Who Are Blind. In International Conference on Human-Computer Interaction. Springer, 209-223.

[16] Adriana De Souza e Silva and Daniel M Sutko. 2011. Theorizing locative technologies through philosophies of the virtual. Communication Theory 21, 1 (2011), 23-42.

[17] Katerina Diamantaki, Charalampos Rizopoulos, Dimitris Charitos, and Nikos Tsianos. 2011. Theoretical and methodological implications of designing and implementing multiuser location-based games. Personal and Ubiquitous Computing 15, 1 (2011), 37-49.

[18] Anders Drachen, Pejman Mirza-Babaei, and Lennart E Nacke. 2018. Games user research. Oxford University Press.

[19] Inger Ekman. 2007. Sound-based gaming for sighted audiencesexperiences from a mobile multiplayer location aware game. In Proceedings of the 2nd audio mostly conference. 148-153.

[20] Magy Seif El-Nasr, Shree Durga, Mariya Shiyko, and Carmen Sceppa. 2015. Data-driven retrospective interviewing (DDRI): a proposed methodology for formative evaluation of pervasive games. Entertainment Computing 11 (2015), 1-19.

[21] Marsha E Fonteyn, Benjamin Kuipers, and Susan J Grobe. 1993. A description of think aloud method and protocol analysis. Qualitative health research 3, 4 (1993), 430-441.

[22] Sarah Holmes. 2019. Play-based 7 interview techniques with young children. Using Innovative Methods in Early Years Research: Beyond the 
Conventional (2019), 92.

[23] Katherine Isbister and Noah Schaffer. 2008. Game usability: Advancing the player experience. CRC press.

[24] Vlasios Kasapakis and Damianos Gavalas. 2015. Pervasive gaming: Status, trends and design principles. Fournal of Network and Computer Applications 55 (2015), 213-236.

[25] Vlasios Kasapakis and Damianos Gavalas. 2017. User-Generated Content in Pervasive Games. Computers in Entertainment (CIE) 16, 1 (2017), 3

[26] Peter Kiefer, Sebastian Matyas, and Christoph Schlieder. 2006. Systematically exploring the design space of location-based games. In 4 th International Conference on Pervasive Computing. 183-190.

[27] Barbara A Kitchenham, David Budgen, and O Pearl Brereton. 2010. The value of mapping studies-A participant-observer case study.. In EASE, Vol. 10. 25-33.

[28] Jason Kurczak, TC Graham, Claire Joly, and Regan L Mandryk. 2011. Hearing is believing: evaluating ambient audio for location-based games. In Proceedings of the 8th International Conference on Advances in Computer Entertainment Technology. ACM, 32.

[29] Steinar Kvale. 2008. Doing interviews. Sage.

[30] Michèle Lamont and Ann Swidler. 2014. Methodological pluralism and the possibilities and limits of interviewing. Qualitative Sociology 37, 2 (2014), 153-171.

[31] Michael Lankes and Barbara Stiglbauer. 2016. GazeAR: Mobile gazebased interaction in the context of augmented reality games. In International Conference on Augmented Reality, Virtual Reality and Computer Graphics. Springer, 397-406.

[32] Jonathan Lazar, Jinjuan Heidi Feng, and Harry Hochheiser. 2017. Research methods in human-computer interaction. Morgan Kaufmann.

[33] Irma Lindt, JAN Ohlenburg, Uta Pankoke-Babatz, and Sabiha Ghellal. 2007. A report on the crossmedia game epidemic menace. Computers in Entertainment (CIE) 5, 1 (2007), 8.

[34] Conor Linehan, Nick Bull, and Ben Kirman. 2013. BOLLOCKS!! Designing pervasive games that play with the social rules of built environments. In International Conference on Advances in Computer Entertainment Technology. Springer, 123-137.

[35] Roderick McCall and Anne-Kathrine Braun. 2008. Experiences of evaluating presence in augmented realities. Psychnology 6, 2 (2008), 157-163.

[36] Rod McCall, Richard Wetzel, Johannes Löschner, and Anne-Kathrin Braun. 2011. Using presence to evaluate an augmented reality location aware game. Personal and Ubiquitous Computing 15, 1 (2011), 25-35.

[37] Timothy Merritt, Christine Linding Nielsen, Frederik Lund Jakobsen, and Jens Emil Grønbæk. 2017. Glowphones: designing for proxemics play with low-resolution displays in location-based games. In Proceedings of the Annual Symposium on Computer-Human Interaction in Play. ACM, 69-81.

[38] Michael D Myers and Michael Newman. 2007. The qualitative interview in IS research: Examining the craft. Information and organization 17, 1 (2007), 2-26.

[39] Lennart E Nacke, Christiane Moser, Anders Drachen, Pejman MirzaBabaei, Andrea Abney, and Zhu Cole Zhenyu. 2016. Lightweight Games User Research for Indies and Non-Profit Organizations. In Proceedings of the 2016 CHI Conference Extended Abstracts on Human Factors in Computing Systems. ACM, 3597-3603.

[40] Tommy Nilsson, Carl Hogsden, Charith Perera, Saeed Aghaee, David J Scruton, Andreas Lund, and Alan F Blackwell. 2016. Applying seamful design in location-based mobile museum applications. ACM Transactions on Multimedia Computing, Communications, and Applications (TOMM) 12, 4 (2016), 56.

[41] Leif Oppermann, Steffen Schaal, Manuela Eisenhardt, Constantin Brosda, Heike Müller, and Silke Bartsch. 2017. Move, Interact, Learn,
Eat-A Toolbox for Educational Location-Based Games. In International Conference on Advances in Computer Entertainment. Springer, 774-794.

[42] A Ant Ozok. 2009. Survey design and implementation in HCI. HumanComputer Interaction: Development Process 253 (2009).

[43] Janne Paavilainen, Hannu Korhonen, Kati Alha, Jaakko Stenros, Elina Koskinen, and Frans Mayra. 2017. The Pokémon GO experience: A location-based augmented reality mobile game goes mainstream. In Proceedings of the 2017 CHI conference on human factors in computing systems. ACM, 2493-2498.

[44] Yen Lina Prasetio, Rendy Wijaya, Michael Pratama Sjah, Michael Ryan Christian, and Andry Chowanda. 2017. Location-based game to enhance player's experience in survival horror game. Procedia computer science 116 (2017), 206-213.

[45] Aung Pyae, Luimula Mika, and Jouni Smed. 2017. Understanding Players' Experiences in Location-based Augmented Reality Mobile Games: A Case of Pokémon Go. In Extended Abstracts Publication of the Annual Symposium on Computer-Human Interaction in Play. ACM, 535-541.

[46] Albert Quek and John See. 2015. Obscura: A mobile game with camera based mechanics. In 2015 Game Physics and Mechanics International Conference (GAMEPEC). IEEE, 21-25.

[47] Yvonne Rogers, Helen Sharp, and Jennifer Preece. 2013. Design de Interação. Bookman Editora.

[48] Gene Rowe and George Wright. 2001. Expert opinions in forecasting: the role of the Delphi technique. In Principles of forecasting. Springer, 125-144.

[49] Jennifer Rowley. 2012. Conducting research interviews. Management research review 35, 3/4 (2012), 260-271.

[50] José Luis González Sánchez, Francisco Luis Gutiérrez Vela, Francisco Montero Simarro, and Natalia Padilla-Zea. 2012. Playability: analysing user experience in video games. Behaviour \& Information Technology 31, 10 (2012), 1033-1054.

[51] Alex Sandham, Tom Ormerod, Coral Dando, Ray Bull, Mike Jackson, and James Goulding. 2011. Scent trails: Countering terrorism through informed surveillance. In International Conference on Engineering Psychology and Cognitive Ergonomics. Springer, 452-460.

[52] Jesse Schell. 2014. The Art of Game Design: A book of lenses. AK Peters/CRC Press.

[53] Ben Shneiderman, Catherine Plaisant, Maxine Cohen, Steven Jacobs, Niklas Elmqvist, and Nicholas Diakopoulos. 2016. Designing the user interface: strategies for effective human-computer interaction. Pearson.

[54] Misha Sra and Chris Schmandt. 2015. Expanding social mobile games beyond the device screen. Personal and Ubiquitous Computing 19, 3-4 (2015), 495-508.

[55] Daniel W Turner III. 2010. Qualitative interview design: A practical guide for novice investigators. The qualitative report 15, 3 (2010), 754-760.

[56] Adrián Juan Verdejo, Katrien De Moor, Istvan Ketyko, Karen Torben Nielsen, Jeroen Vanattenhoven, Toon De Pessemier, Wout Joseph, Luc Martens, and Lieven De Marez. 2010. QoE estimation of a locationbased mobile game using on-body sensors and QoS-related data. In 2010 IFIP Wireless Days. IEEE, 1-5.

[57] Alf Inge Wang, Stian Forberg, and Jon Kjetil Øye. 2016. Knowledge war-a pervasive multiplayer role-playing learning game. In European Conference on Game Based Learning (ECGBL 2016). ACPI, Glasgow, Scotland.

[58] Larry E Wood. 1997. Semi-structured interviewing for user-centered design. interactions 4, 2 (1997), 48-61. 